\title{
The All but Forgotten Mascagni-Sappey Pathway: Learning from Immediate Lymphatic Reconstruction
}

\author{
Anna Rose Johnson, MPH ${ }^{1}$ Miguel G. Bravo, MD ${ }^{1}$ Ted A. James, MD ${ }^{2}$ Hiroo Suami, MD, PhD 3 \\ Bernard T. Lee, MD, MBA, MPH ${ }^{1}$ Dhruv Singhal, MD ${ }^{1}$ \\ ${ }^{1}$ Division of Plastic and Reconstructive Surgery, Department of \\ Surgery, Beth Israel Deaconess Medical Center, Harvard Medical \\ School, Boston, Massachusetts \\ 2 Department of Surgery/Breast Care Center, Beth Israel Deaconess \\ Medical Center, Harvard Medical School, Boston, Massachusetts \\ ${ }^{3}$ Australian Lymphoedema Education, Research and Treatment, \\ Faculty of Medicine and Health Sciences, Macquarie University, \\ Sydney, Australia \\ Address for correspondence Dhruv Singhal, MD, Beth Israel \\ Deaconess Department of Surgery, Division of Plastic and \\ Reconstructive Surgery, 110 Francis Street, Suite 5A, Boston, \\ MA 02215 (e-mail: dsinghal@bidmc.harvard.edu).
}

J Reconstr Microsurg 2020;36:28-31.

\begin{abstract}
\section{Keywords}

- immediate lymphatic reconstruction

- anatomy

- imaging

- lymphatic drainage

Background Upper extremity lymphedema occurs in 25 to $40 \%$ of patients after axillary lymph node dissection (ALND). Immediate lymphatic reconstruction (ILR) or the lymphatic micro- surgical preventative healing approach has demonstrated a significant decrease in postoperative rates of lymphedema (LE) from 4 to $12 \%$. Our objective was to map the Mascagni -Sappey pathway, the lateral upper arm draining lymphatics, in patients undergoing ILR to better characterize the drainage pattern of this lymphosome to the axilla.

Methods A retrospective review of our institutional lymphatic database was conducted and consecutive breast cancer patients undergoing ILR were identified from November 2017 through June 2018. Patient demographics, clinical characteristics, and intraoperative records were retrieved and analyzed.

Results Twenty-nine consecutive breast cancer patients who underwent ILR after ALND were identified. Patients had a mean age of 54 .6years and body mass index (BMI) of $26.6 \mathrm{~kg} / \mathrm{m} 2$. Fluorescein isothiocyanate (FITC) was injected at the medial upper arm and isosulfan blue was injected at the cephalic vein, or lateral upper arm, prior to ALND. After ALND, an average 2.5 divided lymphatics were identified, and a mean 1.2 lymphatics were bypassed. In all patients, divided FITC lymphatics were identified. However, in only three patients (10\%), divided blue lymphatics were identified after ALND.

Conclusion In this study, variable drainage of the lateral upper arm to the axillary bed was noted. This study is the first to provide a description of intraoperative findings, demonstrating variable drainage patterns of upper extremity lymphatics to the axilla. Moreover, we noted that the lateral- and medial-upper arm lymphosomes have mutually exclusive pathways draining to the axilla. Further study of lymphatic anatomy variability may elucidate the pathophysiology of lymphedema development and influence approaches to immediate lymphatic reconstruction.
\end{abstract}

received

March 18, 2019

accepted after revision

June 21, 2019

published online

August 9, 2019
Copyright $\odot 2020$ by Thieme Medical

Publishers, Inc., 333 Seventh Avenue, New York, NY 10001, USA

Tel: +1(212) 584-4662.
DOI https://doi.org/

10.1055/s-0039-1694757. ISSN 0743-684X. 
The lymphatic microsurgical preventative healing approach (LYMPHA) or immediate lymphatic reconstruction (ILR) is performed at time of axillary lymph node dissection (ALND) and reroutes lymphatic flow from divided arm lymphatics into nearby tributaries of the axillary vein. Boccardo et al described this technique in 2009 and has reported a 4\% rate of lymphedema (LE) over a 4-year follow-up period compared with a 25 to $40 \%$ incidence with historic controls. ${ }^{1,2}$ These results have been duplicated at other institutions. ${ }^{3,4}$ The original LYMPHA technique identified arm lymphatics by injecting dye into the medial-upper arm. ${ }^{1}$ However, visualization of divided lymphatics draining the lateral upper arm or the MascagniSappey (M-S) pathway was not attempted. We believe better understanding and mapping of the M-S pathway may have important implications in understanding which patients are at higher risk of developing lymphedema and potential intraoperative implications for surgeons performing ILR.

In 1874 , Sappey proposed that skin territories were drained by a set of lymphatic vessels, the "lymphosome relationship." ${ }^{5-7}$ In the modern era, a work by Suami et al utilizes fluorescent dyes to map lymphatic vessels to their corresponding lymph nodes. ${ }^{8}$ The upper arm has two lymphosomes, medial and lateral. ${ }^{9,10}$ The M-S pathway has historically been used to describe lymphatics draining a territory of the lateral-upper arm. For purposes of this paper, the M-S pathway will be referred to as the "lateral pathway." It has been noted by anatomists to have variable drainage to the supraclavicular/infraclavicular versus axillary lymph nodes. ${ }^{8}$ When the pathway avoids the axillary basin, it serves as an "escape route" outside of the standard operative field and its continued patency would help to prevent LE. ${ }^{6}$ Despite these cadaveric postulations, no intraoperative attempt to identify this pathway has been described.

To comprehensively map all the lymphatics of the upper arm during ILR of the axilla, we chose to inject each lymphosome of the upper arm to ensure that all pathways are accounted for and, if possible, appropriately reconstructed. To differentiate each lymphosome, a different dye was injected into each cutaneous region.

This study is a retrospective review of our experience with the goal of better understanding the anatomy of the M-S pathway.

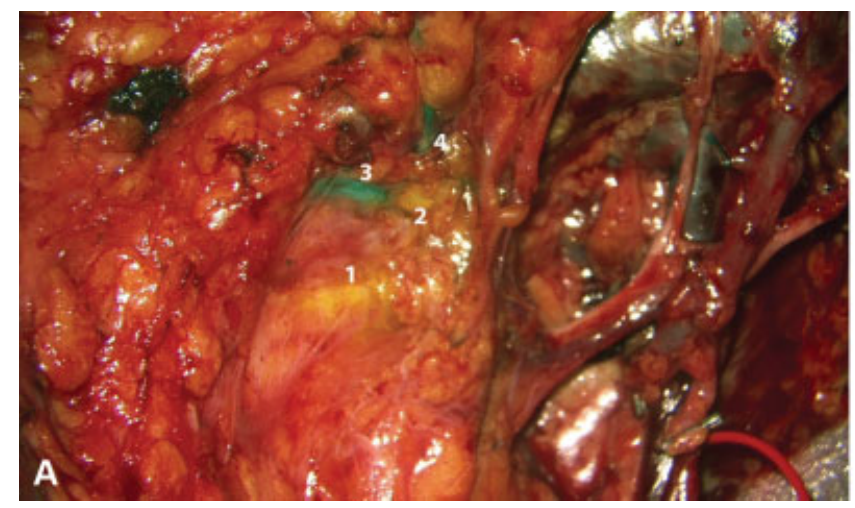

\section{Methods}

A retrospective review of a quality improvement lymphatic surgery database was performed. Patients undergoing immediate lymphatic reconstruction at time of ALND between November 2017 and June 2018 were identified. Institutional Review Board approval was obtained for this study. Patient demographics, clinical characteristics, and intraoperative records were retrieved.

\section{Surgical Technique}

Prior to ALND, fluorescein isothiocyanate (FITC) is injected into the skin and deep tissues of the medial-upper arm as previously described. ${ }^{11}$ To map the M-S pathway, an ultrasound is performed to identify the cephalic vein in the upper arm and $1 \mathrm{cc}$ of isosulfan blue is injected into the skin and deep-soft tissues $4 \mathrm{~cm}$ proximal to the elbow crease over the vein. In our experience, we have found FITC and isosulfan blue to be optimal dyes for visualization in live surgery and advocate for their usage. Following ALND, a Mitaka MM51 microscope (Mitaka Kohki Co., Ltd, Japan) equipped with a $560 \mathrm{~nm}$ filter was utilized for visualization of the divided arm lymphatics.

\section{Results}

A total of 29 eligible patients with de novo, unilateral breast cancer undergoing ILR were identified during the study period. The mean patient age (standard deviation [SD]) was 54.6 years $(\mathrm{SD}=13.5$ years) with a mean body mass index (BMI) of $26.6 \mathrm{~kg} / \mathrm{m}^{2}\left(\mathrm{SD}=4.5 \mathrm{~kg} / \mathrm{m}^{2}\right)$. All patients underwent levels I and II nodal dissection. Mean number of positive lymph nodes and total lymph nodes removed was 1.75 and 14, respectively, during nodal surgery. Also, 55\% patients received neoadjuvant chemotherapy. A mean number of 2.5 lymphatics were identified and a mean of 1.2 lymphatics were bypassed (- Supplementary Table S1; available online only). No intraoperative or postoperative complications were noted. But $100 \%$ of patients demonstrated FITC in the axilla. Three of 29 patients (10\%) demonstrated blue dye in the axilla (-Fig. 1). No lymphatic channels

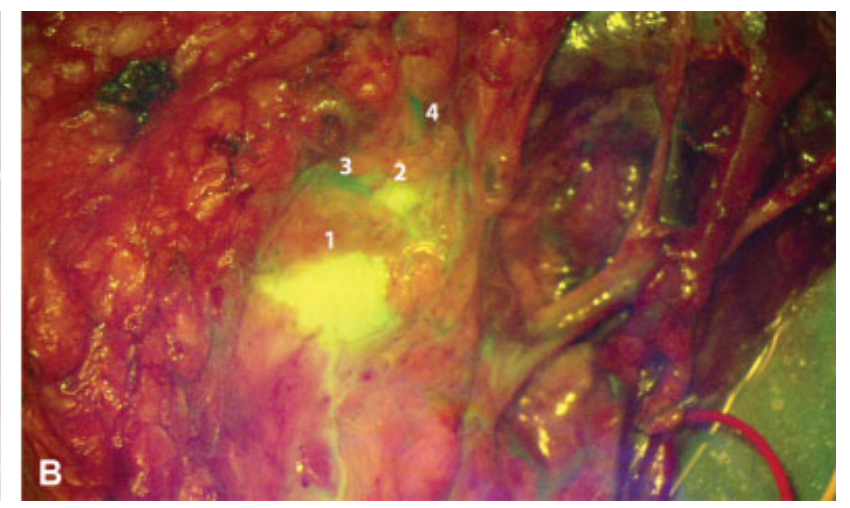

Fig. 1 (A) Following ALND, under white light, blue dye is visualized in lymphatic channels 3 and 4. (B) Upon activation of the $560 \mathrm{~nm}$ filter, FITC was observed in lymphatic channels 1 and 2 . Of note, no crossover of dyes was noted within any lymphatic channel. ALND, axillary lymph node dissection; FITC, fluorescein isothiocyanate. 


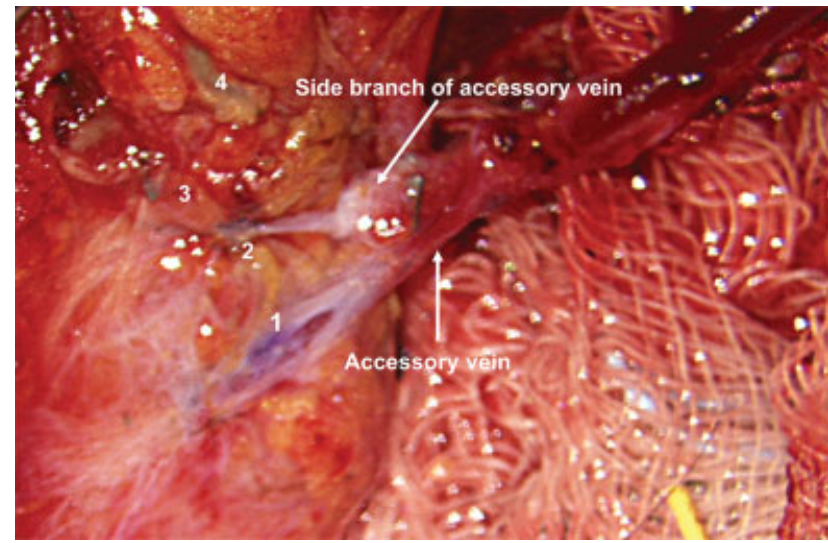

Fig. 2 In cases where both blue and FITC lymphatic channels are identified, attempts are made to bypass both lymphosomes. The two arrows illustrate the accessory vein and a side branch of the accessory vein visualized intraoperatively. FITC, fluorescein isothiocyanate.

demonstrated the concurrent presence of both dyes. There were no differences with respect to patient demographics, neoadjuvant treatment, and nodes removed between patients who demonstrated blue dye in the axilla and those who did not. Twenty-eight of the 29 patients (97\%) were successfully underwent immediate lymphatic reconstruction (-Fig. 2).

\section{Discussion}

Our study found that the Mascagni-Sappey pathway drained to the axillary basin in $10 \%$ of patients undergoing immediate lymphatic reconstruction (denoted by “*” in - Fig. 3). Moreover, in the few cases where the lateral pathway was identified in the axilla, it was distinct from lymphatics draining the medial-upper arm.
Our intraoperative finding that the lateral pathway drains into the axilla in a small subset of the population has significant implications. The lateral pathway has previously been described as a potential "escape route" for the lymphatic system and may explain why, even after regional lymph node radiation (RLNR), and ALND, the rate of lymphedema is approximately 26 to $28 \%$ and not $100 \%{ }^{12-14}$ This simple anatomic variation where the lateral upper arm lymphosome drains into the axillary basin may help predict patients who may have a higher risk of LE after ALND. It may also explain the low but persistent rates of lymphedema following traditional ILR where only lymphatic channels of the medialupper arm lymphosome have been previously bypassed. The fact that our study did not identify any crossover between the lateral- and medial-upper arm, also emphasizes that bypassing divided lymphatic channels of the medial upper arm alone is unlikely to provide any relief to the lateral arm. In the future, lymphatic surgeons may consider ensuring a bypass to each lymphosome identified that drains into the axilla.

\section{Limitations}

There are limitations to the current study. While this study clearly demonstrates our intraoperative findings as they relate to lateral pathway drainage to the axilla, we are unable to qualify if this pathway is simultaneously draining to the supraclavicular/infraclavicular nodes in patients. However, no patient in this study underwent a level III lymph node dissection, precluding any evaluation of drainage to these nodal basins. There were no differences in baseline demographics and preoperative treatment between groups, further supporting that these findings reflect existing anatomic variations. Moreover, we recognize the need for further study in a larger patient population with prolonged follow-up to evaluate its true influence on LE development.

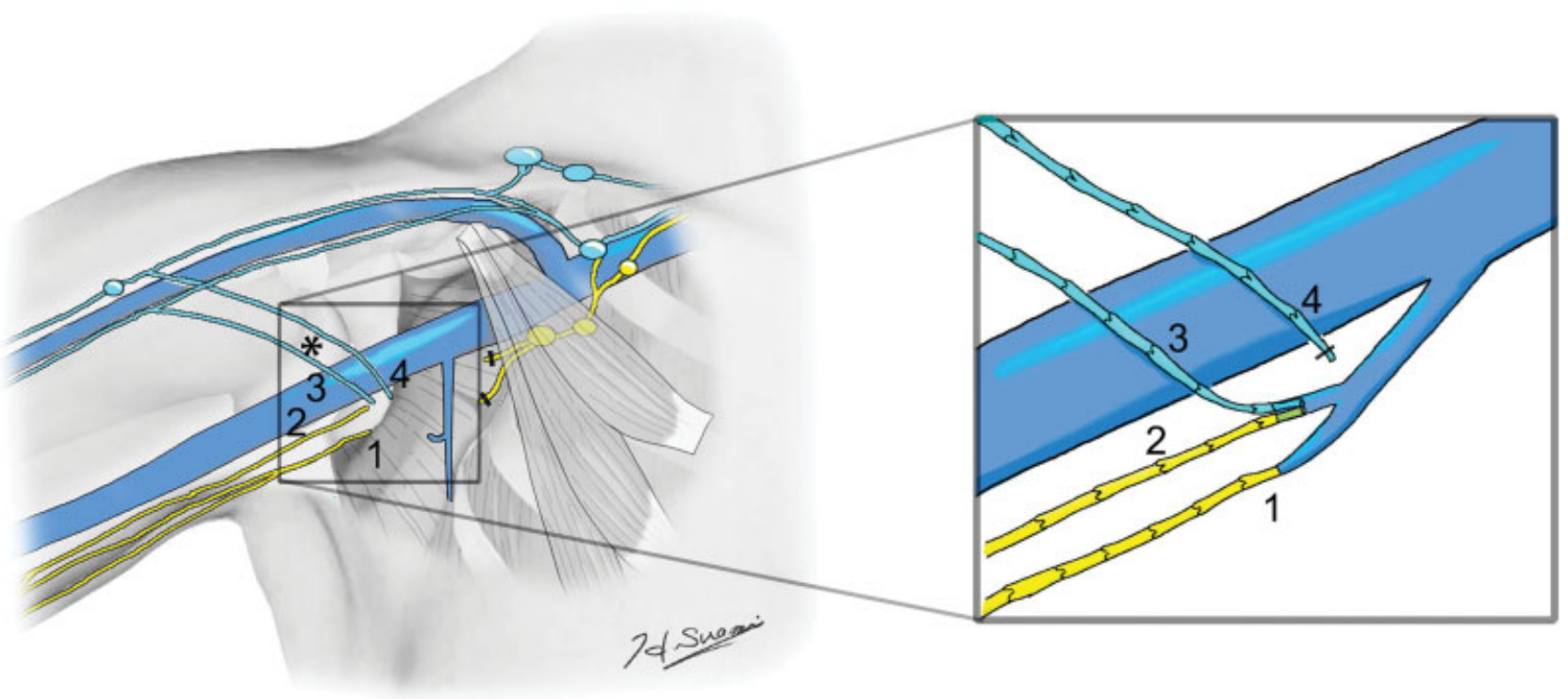

Fig. 3 Schematic illustration of the intraoperative anastomoses performed with corresponding lymphatic channels 1,2,3 and 4. 


\section{Conclusion}

This study is consistent with prior anatomic publications as we have identified variability of the lateral-upper arm drainage. Our study provides the first intraoperative assessment of the lateral pathway in the axilla which may have clinical implications for the lymphatic surgeon performing ILR. Specifically, bypass of the lateral pathway, along with the traditional divided lymphatics of the medial-upper arm, would provide a more comprehensive approach to immediate lymphatic drainage following ALND and may help further reduce the rates of lymphedema. As the field of lymphatic surgery continues to advance and lymphatic anatomy is further elucidated, we anticipate further studies that link anatomic variations with differing rates of lymphedema development.

Funding

None.

\section{Conflict of Interest}

None declared.

\section{References}

1 Boccardo F, Casabona F, De Cian F, et al. Lymphedema microsurgical preventive healing approach: a new technique for primary prevention of arm lymphedema after mastectomy. Ann Surg Oncol 2009;16(03):703-708

2 Boccardo F, Casabona F, De Cian F, et al. Lymphatic microsurgical preventing healing approach (LYMPHA) for primary surgical prevention of breast cancer-related lymphedema: over 4 years follow-up. Microsurgery 2014;34(06):421-424
3 Feldman S, Bansil H, Ascherman J, et al. Single institution experience with lymphatic microsurgical preventive healing approach (LYMPHA) for the primary prevention of lymphedema. Ann Surg Oncol 2015;22(10):3296-3301

4 Hahamoff M, Gupta N, Munoz D, et al. A lymphedema surveillance program for breast cancer patients reveals the promise of surgical prevention. J Surg Res 2018;0(00):S0022-4804(17)30662-5

5 Sappey MPC, Karmanski A, Beau E, Bry A. Anatomie, Physiologie, Pathologie de Vaisseaux Lymphatiques. Paris: Adrain Delahaye, Imprimerie de E. Martinet; 1874

6 Suami H. Lymphosome concept: anatomical study of the lymphatic system. J Surg Oncol 2017;115(01):13-17

7 Suami H, Scaglioni MF. Anatomy of the lymphatic system and the lymphosome concept with reference to lymphedema. Semin Plast Surg 2018;32(01):5-11

8 Suami H, Taylor GI, O'Neill J, Pan WR. Refinements of the radiographic cadaver injection technique for investigating minute lymphatic vessels. Plast Reconstr Surg 2007;120(01):61-67

9 Suami H, Taylor GI, Pan WR. The lymphatic territories of the upper limb: anatomical study and clinical implications. Plast Reconstr Surg 2007;119(06):1813-1822

10 Kubik S. The role of the lateral upper arm bundle and the lymphatic watersheds in the formation of collateral pathways in lymphedema. Acta Biol Acad Sci Hung 1980;31(1-3):191-200

11 Spiguel L, Shaw C, Katz A, et al. Fluorescein Isothiocyanate: A Novel Application for Lymphatic Surgery. Ann Plast Surg 2017;78 (6S, Suppl. 5):S296-S298

12 Johnson AR, Singhal D. Immediate lymphatic reconstruction. J Surg Oncol 2018;118(05):750-757

13 DiSipio T, Rye S, Newman B, Hayes S. Incidence of unilateral arm lymphoedema after breast cancer: a systematic review and metaanalysis. Lancet Oncol 2013;14(06):500-515

14 Suami H, Koelmeyer L, Mackie H, Boyages J. Patterns of lymphatic drainage after axillary node dissection impact arm lymphoedema severity: a review of animal and clinical imaging studies. Surg Oncol 2018;27(04):743-750 